\title{
Spermatogenesis in heterozygotes for Robertsonian chromosomal rearrangements from natural populations of the common shrew, Sorex araneus
}

\author{
S. Garagna, M. Zuccotti, J. B. Searle*, C. A. Redi and P. J. Wilkinson* \\ Dipartimento di Biologia Animale and Centro di Studio per l'Istochimica del CNR, \\ Universitá degli Studi di Pavia, Piazza Botta 10-27100 Pavia, Italy; and *Department of Zoology, \\ University of Oxford, South Parks Road, Oxford OX1 3PS, UK
}

\begin{abstract}
Summary. Twenty-two adult male common shrews were collected from 5 sites in the vicinity of Oxford (UK) close to the zone of hybridization between two karyotypic races. The shrews were subdivided into 3 karyotypic categories: homozygotes, simple Robertsonian heterozygotes (which form one or more trivalents at prophase I of meiosis) and complex Robertsonian heterozygotes (which form a quadrivalent). The ratio of primary spermatocytes to round spermatids was determined from transverse sections of seminiferous tubules, to provide an indication of germ cell death. In no individual was there severe germ cells loss. Homozygotes had the highest mean spermatocyte: spermatid ratio and complex heterozygotes the lowest, but there was substantial individual variation and the differences were not significant. Complex heterozygotes also had a higher proportion of defective seminiferous tubules and lower testis weights than did other categories and it is reasonable to propose that, as a population, complex heterozygotes have reduced fitness relative to other categories on the basis of spermatogenic performance. However, there is no evidence from studies of spermatogenesis that simple Robertsonian heterozygotes are less fit than homozygotes.
\end{abstract}

Keywords: common shrew; Robertsonian rearrangements; spermatogenesis; Robertsonian heterozygotes

\section{Introduction}

Intraspecific karyotypic variation in mammals commonly involves presence or absence of particular chromosomal (structural) rearrangements known as Robertsonian (or centric) fusions. In these rearrangements, a pair of acrocentric (single-armed) chromosomes fuse at their centromeres to form a single (bi-armed) metacentric chromosome. In several species, most notably the house mouse (Mus musculus) and common shrew (Sorex araneus), there is subdivision into a large number of karyotypic races each with a characteristic set of Robertsonian metacentrics in a homozygous state. When such races make contact and hybridize, Robertsonian heterozygotes are produced. If the races differ such that for each Robertsonian metacentric found in one race the homologous ancestral acrocentrics characterize the other, then 'simple' Robertsonian heterozygotes (which form one or more trivalents at prophase I of meiosis) will be produced. Alternatively, the different sets of Robertsonian metacentrics in the two races may include certain metacentrics which differ but which have one arm in common. In this case, double or multiple Robertsonian heterozygotes with monobrachial homology would be expected on hybridization. Such 'complex heterozygotes' are expected to form chain or ring configurations of four or more elements at prophase I of meiosis.

Karyotypic heterozygotes are generally expected to be less fertile than homozygotes (White, 1978). Evidence from a variety of mammals, but particularly laboratory mice, suggests that this is 
the case for Robertsonian heterozygotes in mammals (reviews: Gropp \& Winking, 1981; Redi \& Capanna, 1988; Searle, 1988). There is a higher frequency of anaphase I nondisjunction associated with trivalents and particularly longer configurations than recorded for bivalents. This nondisjunction ultimately results in the production of aneuploid embryos which usually die during gestation, thus leading to smaller litter sizes. Male Robertsonian heterozygotes may additionally suffer from substantial germ cell death. In laboratory mice, complex Robertsonian heterozygotes which are expected to form chain configurations of four or more elements at prophase I may suffer complete spermatogenic arrest (Winking, 1980).

In contrast to such studies on laboratory mice, there have been few studies on fertility of Robertsonian heterozygotes from natural populations of mammals. Yet studies of this sort are essential for a real understanding of the origin and maintenance of Robertsonian variation in nature. In particular, the structure of karyotypic hybrid zones and the extent to which they form a barrier to gene flow between races is partly determined by the degree of selection against heterozygotes (Barton \& Hewitt, 1985). Wild mice with simple Robertsonian heterozygosity have been found to have very low anaphase I nondisjunction frequencies (Winking, 1986; Winking et al., 1988 ) but little is known about spermatogenesis in such mice. A low frequency of anaphase I nondisjunction was also found in wild-caught simple Robertsonian heterozygotes of the common shrew (Sorex araneus) from a region of polymorphism associated with the hybrid zone between the 'Oxford' and 'Hermitage' Robertsonian races in the vicinity of Oxford, UK (Searle, 1984a, 1986a, b). By sampling common shrews from the same region, we present, for the first time, a detailed study of the impact of Robertsonian heterozygosity (both 'simple' and 'complex') on spermatogenesis in individuals from natural populations of mammals.

\section{Materials and Methods}

The shrews. Twenty-two adult male shrews were collected from 5 sites in the vicinity of Oxford (UK): Bletchingdon (grid reference 451/217), Cothill (446/199), East Hendred (445/185), Hodcott Down (448/182) and Eling (451/175). Bletchingdon and Cothill are sites characterized by shrews of the Oxford karyotypic race, while East Hendred, Hodcott Down and Eling are situated within the hybrid zone between the Oxford and Hermitage races (Searle, 1986b). Most of the animals were collected during May and June 1984 (Table 1). These months represent the peak of the breeding seasons; the testes are fully grown and functional (Brambell, 1935).

After capture the shrews were maintained singly indoors but with natural illumination. They were killed within 8 days of collection, the testes were weighed and the left testis was fixed in Bouin's solution. Sperm counts, made on the right epididymis by a method based on that of Searle \& Beechey (1974), are expressed as total number per caput epididymidis. The karyotype of each animal was determined from routine bone marrow preparations, stained by a G-banding method (Searle, 1986b).

The animals were subdivided into three categories on the basis of karyotype: homozygotes, 'simple' heterozygotes and 'complex' heterozygotes. In the description of the karyotype, the nomenclature of Halkka et al. (1974), as clarified by Fredga \& Nawrin (1978), is used. In this nomenclature the letters $a$ - $u$ refer to chromosome arms; arms $j, k, l, n, o, p$, $q$ and $r$ displayed Robertsonian variation in the populations sampled (i.e. these chromosomes arms occurred as acrocentrics or attached to another arm as a metacentric). Among the homozygotes examined, 'Oxford race homozygotes' were homozygous for metacentrics $k q$, no and pr, 'Hermitage race homozygotes' were homozygous for metacentric ko (chromosome arms $n, p, q$ and $r$ occurred as acrocentrics) while individuals with an 'all acrocentric karyotype' were homozygous acrocentric for $k, n, o, p, q$ and $r$. Simple heterozygotes were heterozygous for one or more of the Oxford race arm conbinations $k q$, no and pr or the Hermitage race arm combination $k o$ and were expected to form trivalents (e.g. $n-n o-o$ ) at prophase I of meiosis. One individual was heterozygous for arm combination $j l$, which otherwise occurred in a homozygous metacentric state. The karyotype of the complex heterozygotes examined included both Hermitage metacentric $k o$ and Oxford race metacentric no. It was expected that these complex heterozygotes formed a quadrivalent $(k-k o-o n-n)$ at prophase I of meiosis. Shrews 906 and 907 were also simple heterozygotes for the Oxford race metacentric $p r$.

Histology, staining and cell counting. A standard histological protocol was adopted to obtain $7 \mu \mathrm{m}$ sections. These were stained by the periodic acid-Schiff reaction and counterstained with haematoxylin.

Truly transverse cross-sections of seminiferous tubules were selected for scoring by light microscopy. Five sections for each stage of the seminiferous epithelium cycle (Fig. 1) were examined and the numbers of primary spermatocytes and round spermatids (i.e. spermatids from step 1 to step 6) were scored. All the cell counts were performed under a $\times 100$ oil-immersion objective and the Abercrombie (1946) correction was applied. Cell counts are always expressed 


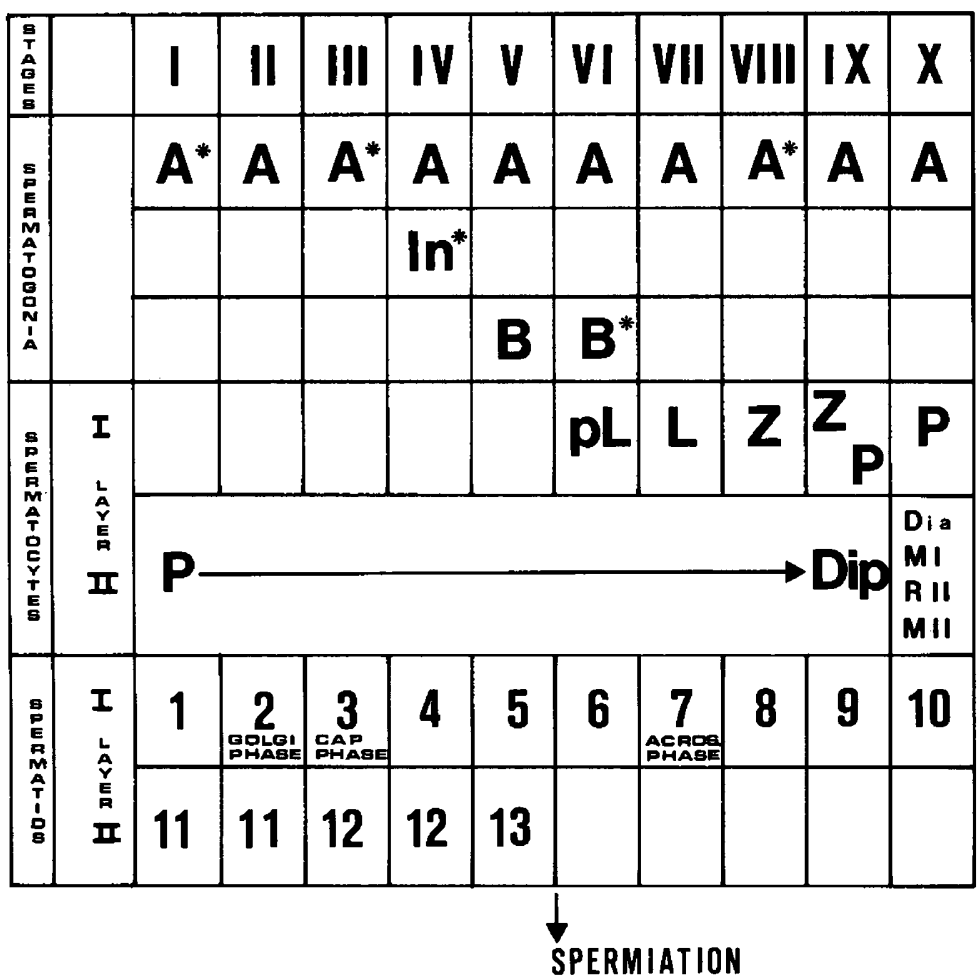

Fig. 1. Schematic representation of the typical cell associations for each stage of the seminiferous epithelium $(\mathrm{I}-\mathrm{X})$. These cell associations were established on the basis of 100 transverse tubule cross-sections each from two shrews of standard homozygous karyotype collected in Eling (Shrew 875 and Shrew 878: Table 1). 'A', 'In' and 'B' indicate the A, Intermediate and B spermatogonia, respectively, while * indicates the occurrence of mitotic divisions. pL: preleptotene, L: leptotene, $\mathrm{Z}$ : zygotene, P: pachytene, Dip: diplotene and Dia: diakinesis stages of primary spermatocytes. RII: resting secondary spermatocytes. MI and MII: first and second mitotic divisions, respectively. The numbers 1-13 indicate the steps of spermatid differentiation.

per cross-section. (Similar results were obtained when cell counts per 100 Sertoli cells or per $100 \mu \mathrm{m}$ of tubular perimeter were calculated.)

The cell counts were used to calculate the mean ratio of 'primary spermatocytes:round spermatids' per animal (Table 1) based on the relative occurrence of these two cell types at stages I-VI of the seminiferous epithelium cycle as defined in Fig. 1. The expected ratio of these cell types is 1:4, if spermatogenesis proceeds perfectly. Considering either the complete data set or the individual karyotypic categories (homozygotes, simple heterozygotes, complex heterozygotes), this ratio did not vary significantly between animals according to date of death. Therefore all individuals are considered to be fully comparable with each other despite slight differences in the date on which they were killed.

The spermatocyte:spermatid ratio provides an indication of overall cell death between the primary spermatocyte and round spermatid stages in the testis. Final sperm production may also be influenced by the distribution of such cell death among tubules. Therefore, the proportion of tubules which had a spermatocyte:spermatid ratio of less than $\mathrm{l}: 3$ (arbitrarily defined as 'defective tubules') was calculated for each individual.

\section{Results}

Table 1 presents the testis weights, sperm counts, mean spermatocyte:spermatid ratios and proportion of defective tubules for each individual collected, in relation to karyotype. The overall impression from this material is that in no case was spermatogenesis severely impaired. There were 
Table 1. Reproductive characteristics of wild-caught common shrews from the Oxford area, in relation to karyotype

\begin{tabular}{|c|c|c|c|c|c|c|c|}
\hline Shrew & $\begin{array}{c}\text { Date } \\
\text { killed* }\end{array}$ & $\begin{array}{l}\text { Site of } \\
\text { capture }\end{array}$ & Karyotype $\dagger$ & $\begin{array}{l}\text { Sperm count } \\
\left(\times 10^{-6}\right)\end{array}$ & $\begin{array}{l}\text { Weight of } \\
\text { combined } \\
\text { testes } \\
(\mathrm{mg})\end{array}$ & $\begin{array}{l}\text { Mean } \\
\text { s'cyte: } \\
\text { s'tid } \\
\text { ratio }\end{array}$ & $\begin{array}{c}\text { Defective } \\
\text { tubules } \\
(\%)_{*}^{*}\end{array}$ \\
\hline & & & Homozygotes & & & & \\
\hline 938 & 14 June & Bletchingdon & $\mathbf{M} j l, k q, n o, p r$ & 7.90 & 208 & $1: 3 \cdot 40$ & $44 \cdot 4$ \\
\hline 936 & 14 June & Bletchingdon & (Oxford) & $16 \cdot 25$ & 271 & $1: 3 \cdot 27$ & $48 \cdot 1$ \\
\hline 841 & 27 April & Cothill & & - & - & $1: 3 \cdot 88$ & $27 \cdot 7$ \\
\hline 910 & 30 May & Hodcott Down & $\mathrm{M} j l \mathrm{~A} k, n, o, p, q, r$ & 12.55 & 203 & $1: 3 \cdot 57$ & $33 \cdot 3$ \\
\hline 908 & 4 June & Hodcott Down & (all acrocentric & $6 \cdot 20$ & 217 & $1: 3 \cdot 34$ & 1.4 \\
\hline 904 & 28 May & Hodcott Down & individuals) & $11 \cdot 05$ & 235 & $1: 2 \cdot 60$ & $92 \cdot 6$ \\
\hline 899 & 4 June & Hodcott Down & & 8.55 & 304 & $1: 3 \cdot 84$ & $38 \cdot 8$ \\
\hline 878 & 16 May & Eling & $\mathrm{M} j l, k o \mathrm{~A} n, p, q, r$ & $11 \cdot 90$ & 211 & $1: 3 \cdot 88$ & $22 \cdot 2$ \\
\hline \multirow[t]{2}{*}{875} & 14 May & Eling & (Hermitage) & 9.65 & 231 & $1: 3 \cdot 50$ & $36 \cdot 0$ \\
\hline & & & Means & $10 \cdot 51$ & $\overline{235 \cdot 0}$ & $\overline{1: 3 \cdot 48}$ & $\overline{38 \cdot 3}$ \\
\hline & & & Simple heterozygotes & & & & \\
\hline 902 & 31 May & Hodcott Down & $\mathrm{M} j l \mathrm{H} n o \mathrm{~A} k, p, q, r$ & $10 \cdot 20$ & 239 & $1: 2.93$ & $81 \cdot 5$ \\
\hline 913 & 29 May & Hodcott Down & $\mathrm{M} j l \mathrm{H} p r \mathrm{~A} k, n, o, q$ & 13.05 & 234 & $1: 3.40$ & $50 \cdot 0$ \\
\hline 911 & 30 May & Hodcott Down & $\mathrm{M} j l \mathrm{H} k o \mathrm{~A} n, p, q, r$ & $10 \cdot 80$ & 260 & $1: 2.69$ & $91 \cdot 3$ \\
\hline 901 & 31 May & Hodcott Down & & $18 \cdot 25$ & 307 & $1: 2 \cdot 61$ & $77 \cdot 7$ \\
\hline 870 & 15 May & Eling & & $11 \cdot 20$ & 217 & $1: 3 \cdot 80$ & $5 \cdot 5$ \\
\hline 868 & 16 May & Eling & & 12.05 & 243 & $1: 3.67$ & $33 \cdot 3$ \\
\hline 909 & 31 May & Hodcott Down & $\mathrm{H} j l, n o \mathbf{A} k, p, q, r$ & 19.95 & 297 & 1:3.04 & $66 \cdot 6$ \\
\hline 900 & 30 May & Hodcott Down & $\mathrm{M} j l \mathrm{H} n o, p r \mathrm{~A} k, q$ & $17 \cdot 70$ & 338 & $1: 3.91$ & $27 \cdot 7$ \\
\hline \multirow[t]{2}{*}{930} & 11 June & Cothill & $\mathrm{M} j l \mathrm{H} k q, n o, p r$ & $9 \cdot 15$ & 268 & $1: 3 \cdot 42$ & $44 \cdot 4$ \\
\hline & & & Means & $\overline{13.59}$ & $\overline{267 \cdot 0}$ & $1: 3 \cdot 27$ & $\overline{53 \cdot 1}$ \\
\hline \multirow{6}{*}{$\begin{array}{r}2095 \\
702 \\
906 \\
907\end{array}$} & & & Complex heterozygotes & & & & \\
\hline & $10 \mathrm{May}$ & East Hendred & $\mathrm{M} j l \mathrm{H} k o, n o \mathrm{~A} p, q, r$ & - & 248 & $1: 3 \cdot 39$ & $16 \cdot 7$ \\
\hline & 7 April & East Hendred & & - & $176 \S$ & $1: 2.79$ & $50 \cdot 0$ \\
\hline & $30 \mathrm{May}$ & Hodcott Down & $\mathrm{M} j l \mathrm{H} k o, n o, p r \mathrm{Aq}$ & $11 \cdot 15$ & $192^{\circ}$ & $1: 2.94$ & $73 \cdot 0$ \\
\hline & 29 May & Hodcott Down & & 6.80 & 170 & $1: 2.52$ & $92 \cdot 6$ \\
\hline & & & Means & 8.98 & 196.5 & $1: 2.91$ & $58 \cdot 1$ \\
\hline
\end{tabular}

*Year 1984 except for No. 2095 (year 1988) and No. 702 (year 1983).

†Only variable chromosome arms recorded (see Searle, 1986b). M: homozygous metacentric, $\mathrm{H}$ : heterozygous, A: homozygous acrocentric.

¥Defective tubules have a spermatocyte:spermatid ratio of less than $1: 3$.

$\S$ Twice weight of right testis.

no cases of complete spermatogenic arrest, as recorded for an XXY adult male common shrew (Searle, 1984b), or partial arrest as recorded for an XY/XYY mosaic adult male (Searle \& Wilkinson, 1986). For those individuals (caught in May 1981 and June 1984, respectively), weights of the combined testes were $5 \mathrm{mg}$ (approximately) and $112 \mathrm{mg}$, respectively, and sperm count for the XY/XYY mosaic was $0.3 \times 10^{6}$ (no spermatozoa were produced in the XXY male). For the shrews described in the present paper the weights of the combined testes varied between 170 and $338 \mathrm{mg}$ and sperm counts between $6.2 \times 10^{6}$ and $20.0 \times 10^{6}$ (Table 1). On the basis of criteria used for laboratory mice, these sperm counts indicate full fertility (mice are considered to be infertile when the sperm count is below 10\% of normal: Searle \& Beechey, 1974).

The mean spermatocyte:spermatid ratios varied between 2.52 and 3.91 , overall. Again this indicates that spermatogenesis was never seriously defective. Also, the ratio was invariably less than the 'perfect' spermatocyte:spermatid ratio of 1:4. There was substantial variation in this ratio between individuals of the same karyotype from the same site. 
Nine individuals had a homozygous karyotype. The Oxford race homozygotes were collected from sites well to the north of the Oxford-Hermitage hybrid zone, while all-acrocentric individuals and Hermitage-race individuals were collected from sites within the hybrid zone. The spermatocyte and spermatid populations were very similar in all three types of homozygote (Fig. 2a) and, with the exception of one individual (No. 904), the mean spermatocyte:spermatid ratio varied over the range 3.3-3.9 (Table 1). Shrew 904 had a ratio of $2 \cdot 6$. This individual was also the only individual among the homozygotes in which the proportion of 'defective' tubules was $50 \%$ or greater. In fact, nearly all tubules were defective in this individual (Table 1).

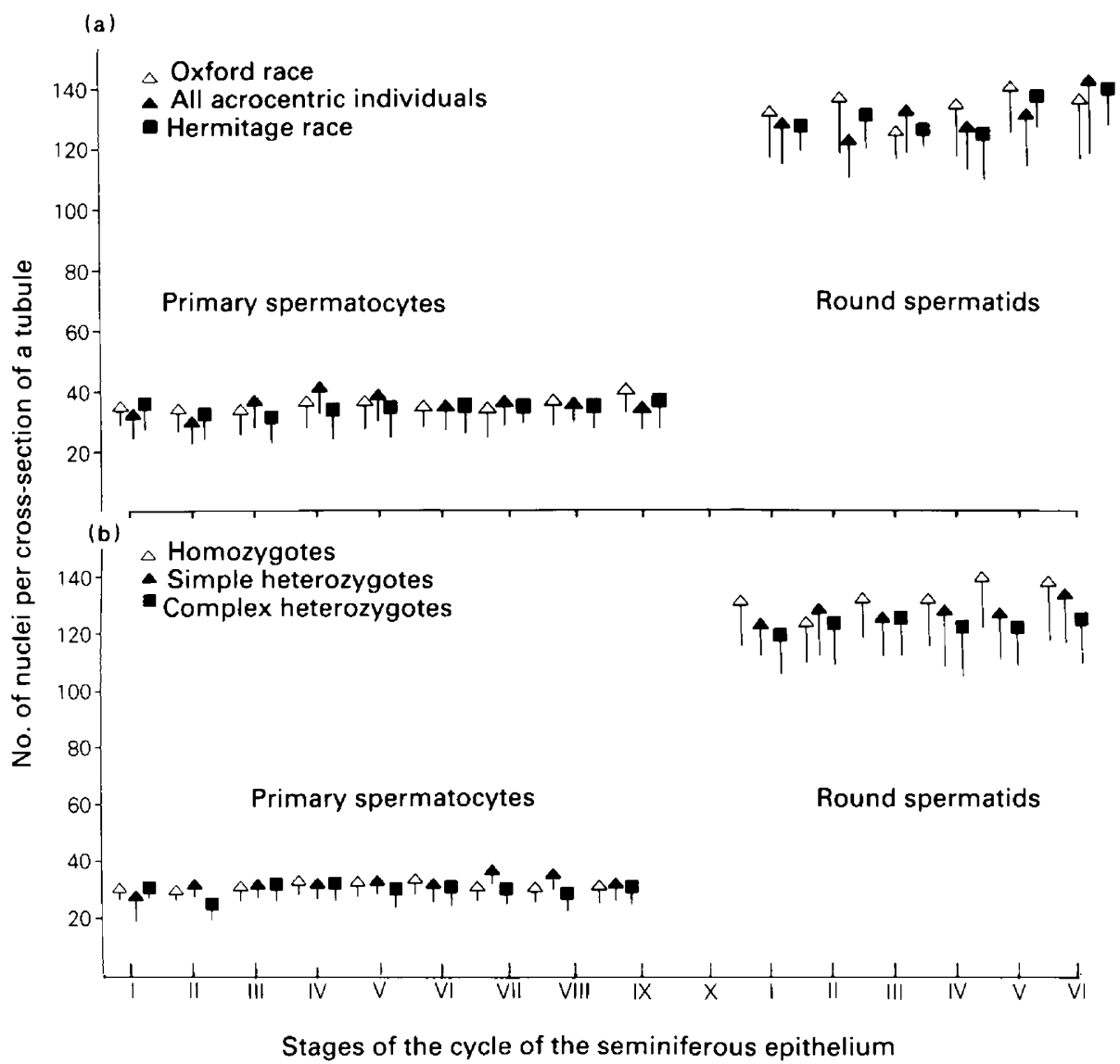

Fig. 2. Kinetics of spermatogenesis in terms of populations of spermatocytes and spermatids (means and standard deviations) for different karyotypic homozygotes (a) and homozygotes, simple heterozygotes and complex heterozygotes (b).

Nine simple heterozygotes were examined, all but one from hybrid zone sites. Individual variation in spermatocyte:spermatid ratio was greater among these individuals than for homozygotes, but the extremes were again 2.6 and 3.9 (Table 1). Overall, the mean spermatocyte:spermatid ratio was lower than in homozygotes and as many as 5 out of 9 individuals had $50 \%$ or more defective tubules (Table 1). In contrast, testis weights and sperm counts were higher, on average, than in homozygotes.

The four complex heterozygotes were hybrids. They had the lowest testis weights and spermatocyte:spermatid ratios, on average (Table 1). Also, 3 out of 4 individuals had $50 \%$ or more defective tubules (Table 1). 
Therefore, considering mean spermatocyte:spermatid ratios, there is a trend of increasing germ cell death with complexity of karyotype, with least cell loss in homozygotes and most in complex heterozygotes (Table 1). A similar trend is apparent for proportion of defective tubules (Table 1). However, in reiteration of earlier observations, the data indicate that spermatogenesis was never grossly impaired in individuals of any karyotypic category. Figure 2(b) shows the mean numbers of spermatocytes and spermatids in cross-sections at each stage of the seminiferous epithelium for homozygotes, simple heterozygotes and complex heterozygotes. While there were, on average, most round spermatids in tubule cross-sections of homozygotes and least in complex heterozygotes for almost all epithelial stages, the differences in degree of germ cell death between karyotypic categories were marginal in comparison with the differences previously described between homozygote and complex Robertsonian heterozygote mice (Fig. 11 in Gropp et al., 1982). There was also considerable individual variation within each karyotypic category and the difference in mean spermatocyte:spermatid ratio between categories is not significant $\left(\mathrm{F}_{2,19}=2 \cdot 39, P>0.05\right)$.

Testis weights did vary significantly between homozygotes, simple heterozygotes and complex heterozygotes $\left(\mathrm{F}_{2,18}=5.13, P<0.05\right)$. In particular, the simple heterozygotes had significantly heavier testes than did complex heterozygotes (Scheffe's test, $P<0.05$ ). The results were similar for sperm counts, with highest counts in simple heterozygotes and lowest in complex heterozygotes (Table 1). There was a significant correlation between testis weight and sperm count $(r=0.64$, $t=3 \cdot 45,17$ d.f., $P<0 \cdot 01$ ).

Overall, there was no correlation between the spermatocyte:spermatid ratio and testis weight. However, among the individual karyotypic categories, there was a positive correlation between these measures for the complex heterozygotes $(r=0.96, t=4.99,2$ d.f., $P<0.05)$. Testis weight is low when there is substantial germ cell death in the mouse (Searle \& Beechey, 1974) and shrew (Searle, 1984b; Searle \& Wilkinson, 1986). It is perhaps not surprising therefore that the correlation between spermatocyte:spermatid ratio and testis weight holds for the karyotypic category in which there is the greatest germ cell death.

\section{Discussion}

Although there was substantial variation in the reproductive characteristics of the 22 wild adult male common shrews examined in this study, there was no evidence that this variation had any effect on the relative fertility of these particular individuals. In our discussion of the results we are therefore looking for indications of possible predisposition to spermatogenic impairment among the 3 karyotypic categories.

As measured by the ratio of primary spermatocytes to round spermatids, there was no significant difference in the extent of germ cell death between the different karyotypic categories. However, both in terms of this ratio and the proportion of 'defective' tubules, there were indications that spermatogenesis proceeded more perfectly in the homozygotes than in the heterozygotes, particularly the complex heterozygotes. This may reflect a higher frequency of chromosome pairing abnormalities at pachytene in karyotypic heterozygotes. Such abnormalities may lead to germ cell death due to inappropriate gene expression (Forejt, 1982; Burgoyne \& Baker, 1984). It should be noted that we did not examine germ cell death during the stages of elongation and maturation of spermatids due to the difficulty of counting non-spherical nuclei. However, sperm counts do not indicate substantial losses at these stages in heterozygotes relative to homozygotes.

In the simple heterozygotes, any small reduction in sperm production due to germ cell loss was outweighed by the larger testis size. The sperm count was higher on average in simple heterozygotes than homozygotes, presumably as a reflection of this greater testis size (sperm count and testis size are highly correlated). Any discrepancy in testis size between simple heterozygotes and homozygotes could reflect genic heterozygosity. Searle (1988) discusses how heterosis may evolve in simple Robertsonian heterozygotes. In contrast, complex heterozygotes had the lowest testicular weights 
and sperm counts and, as in the case of other interracial hybrids (Barton \& Hewitt, 1985), genic heterozygosity may be disadvantagous in this case.

Thus, amongst common shrews from the vicinity of the Oxford-Hermitage hybrid zone, there is no evidence that simple Robertsonian heterozygotes have a reduced fitness relative to homozygotes, on the basis of spermatogenic performance. Nor is there any great fertility reduction in such heterozygotes relative to homozygotes due to anaphase I nondisjunction (Searle, 1984a, 1986a). These results are of importance because the ease with which a Robertsonian metacentric will spread into populations characterized by the homologous twin-acrocentric state will depend on the relative fertility of homozygotes and simple Robertsonian heterozygotes. Clearly, with the fertility of simple Robertsonian heterozygotes relatively high, the spread of the metacentric may not be so greatly impeded. The situation may be different for the spread of a metacentric into populations already characterized by a different metacentric with monobrachial homology, as the complex heterozygotes produced may be expected to have a poorer spermatogenic performance than homozygotes.

It is important to consider whether this poorer spermatogenic performance of complex heterozygotes compared with homozygotes may be substantial enough to reduce fertility, and hence fitness. As already discussed it is unlikely that the individuals with a complex heterozygote karyotype actually examined had a reduced fertility relative to other individuals. However, from the whole data set, it is apparent that there was considerable individual variation. In man, there is also substantial variation in germ cell loss between individuals such that heterozygotes for the Robertsonian metacentric $t(14 ; 21)$ may range from fully fertile to sterile (Rosenmann et al., 1985). If the poorer spermatogenic performance of complex heterozygotes manifests itself as a greater predisposition to sterility, this will be of significance. Thus, if the proportion of sterile males is only marginally greater among the complex heterozygous shrews from the hybrid zone between the Oxford and Hermitage races than among homozygous Oxford and Hermitage race individuals, it could still be sufficient to influence the extent of hybridization between these races.

There is little information on other possible sources of fertility reduction in complex Robertsonian heterozygotes. However, preliminary microdensitometry studies of sperm DNA indicated a higher frequency of anaphase I nondisjunction in complex Robertsonian heterozygotes than homozygotes (Searle, 1988). Frequency data from the field strongly suggest that complex Robertsonian heterozygotes do have a lower fitness than homozygotes (Searle, 1986b) and the fertility data presented in this paper are consistent with this interpretation.

We thank Dr J. R. Clarke and Dr A. E. Douglas for comments on the paper; Dr J. R. Clarke for laboratory facilities; and S. J. Mercer for collecting and karyotyping Shrew 2095. This work was supported by the Italian CNR 'Bilateral Project N. 87.00123.04' and the Royal Society of London.

\section{References}

Abercrombie, M. (1946) Estimation of nuclear population microtome sections. Anat. Rec. 94, 238-248.

Barton, N.H. \& Hewitt, G.M. (1985) Analysis of hybrid zones. Ann. Rev. Ecol. Syst. 16, 113-148.

Brambell, F.W.R. (1935) Reproduction in the common shrew (Sorex araneus Linnaeus). II. Seasonal changes in reproductive organs of the male. Phil. Trans. $R$. Soc. Lond. B 225, 51-62

Burgoyne, P.S. \& Baker, T.G. (1984) Meiotic pairing and gametogenic failure. In Controlling Events in Meiosis (38th Symp. Soc. exp. Biol.), pp. 349-362. Eds C. W. Evans \& H. G. Dickinson. Company of Biologists, Cambridge.

Forejt, J. (1982) X-Y involvement in male sterility caused by autosome translocations - a hypothesis. In Genetic Control of Gamete Production and Function, pp. 135-151. Eds P. G. Crosignani, B. L. Rubin \& M. Fraccaro. Grune \& Stratton, New York.

Fredga, K. \& Nawrin, J. (1977) Karyotype variability in Sorex araneus L. (Insectivora, Mammalia). Chromosomes Today 6, 153-161.

Gropp, A. \& Winking, H. (1981) Robertsonian translocations: cytology, meiosis, segregation patterns and biological consequences of heterozygosity. Symp. zool. Soc. Lond. 47, 141-181.

Gropp, A., Winking, H. \& Redi, C. (1982) Consequences of Robertsonian heterozygosity: segregational impairment of fertility versus male-limited sterility. In Genetic Control of Gamete Production and Function, pp. 115-134. Eds P. G. Crosignani, B. L. Rubin \& M. Fraccaro. Grune \& Stratton, New York. 
Halkka, L., Halkka, O., Skarén, U. \& Söderlund, V. (1974) Chromosome banding pattern in a polymorphic population of Sorex araneus from northeastern Finland. Hereditas 76, 305-314.

Redi, C.A. \& Capanna, E. (1988) Robertsonian heterozygotes in the house mouse and the fate of their germ cells. In The Cytogenetics of Mammalian Autosomal Rearrangements, pp. 315-359. Ed. A. Daniel. Liss, New York.

Rosenmann, A., Wahrman, J., Richler, C., Voss, R., Persitz, A. \& Goldman, B. (1985) Meiotic association between the $\mathrm{XY}$ chromosomes and unpaired autosomal elements as a cause of human male sterility. Cytogenet. Cell Genet. 39, 19-29.

Searle, A.G. \& Beechey, C.V. (1974) Sperm count, egg-fertilization and dominant lethality after Xirradiation of mice. Mutation Res. 22, 63-72.

Searle, J. B. (1984a) Nondisjunction frequencies in Robertsonian heterozygotes from natural populations of the common shrew, Sorex araneus L. Cytogenet. Cell Genet. 38, 265-271.

Searle, J.B. (1984b) A wild common shrew (Sorex araneus) with an XXY sex chromosome constitution. $J$. Reprod. Fert. 70, 353-356.

Searle, J.B. (1986a) Meiotic studies of Robertsonian heterozygotes from natural populations of the common shrew, Sorex araneus L. Cytogenet. Cell Genet. 41, 154-162.

Searle, J.B. (1986b) Factors responsible for a karyotypic polymorphism in the common shrew, Sorex araneus. Proc. R. Soc. Lond. B 229, 277-298.

Searle, J.B. (1988) Selection and Robertsonian variation in nature: the case of the common shrew. In The Cytogenetics of Mammalian Autosomal Rearrangements, pp. 507-531. Ed. A. Daniel. Liss, New York.

Searle, J.B. \& Wilkinson, P.J. (1986) The XYY condition in a wild mammal: an $X Y / X Y Y$ mosaic common shrew (Sorex araneus). Cytogenet. Cell Genet. 41, 225-233.

White, M.J.D. (1978) Modes of Speciation. Freeman, San Francisco.

Winking, H. (1980) Cytogenetic and histological observations in sterile males with Robertsonian translocations. Cytogenet. Cell Genet. 27, 213.

Winking, H. (1986) Some aspects of Robertsonian karyotypic variation in European wild mice. Current Topics Microbiol. Immunol. 127, 68-74.

Winking, H., Dulić, B. \& Bulfield, G. (1988) Robertsonian karyotype variation in the European house mouse. Mus musculus. Z. Saugetierk. 53, 148-161.

Received 19 December 1988 